\title{
Grip strength is associated with nutritional status and energy intake in healthy community living older adults
}

\author{
J. M. Jones, G. Baer and H. I. M. Davidson \\ School of Health Sciences, Queen Margaret University, Edinburgh EH21 6UU, UK
}

The UK has an ageing population and this will place large demands on future health and social care systems ${ }^{(1)}$. In an attempt to minimise this increase in expenditure, it is important to encourage the ageing population to optimise their health and ability to live independently. The functional marker grip strength, determined by handgrip dynamometry (HGD), has been shown to be a predictor of all cause mortality ${ }^{(2)}$, disability ${ }^{(3)}$ and thus dependency and in older adults. While dietary intake and nutritional supplementation has been shown to improve function in the rehabilitation phase of care ${ }^{(4)}$, the association dietary intake has with nutritional and functional status has not be fully investigated in the community dwelling older adult population. Handgrip may provide an indication of health status in this ever expanding population. The aim of the study was to determine whether an association exists between energy intake, nutritional status and HGD.

Healthy, cognitively intact, community dwelling adults aged $65+$ years were recruited from community networks. Energy intake $(1 \mathrm{kcal}=4.184 \mathrm{~kJ})$ was recorded by a 7-d self-administered unweighed food diary. Anthropometric measurements (weight, height, BMI, waist circumference (WC), tricep skin-fold thickness (TSF) and arm muscle circumference (AMC)), were measured using standard methodology. HGD was measured using a Takei grip strength dynamometer in the non-dominant arm. BMI was compared to age-specific norms, and TSF, AMC, WC and HGD were compared to gender-specific norms. Correlation analysis was used to identify associations between variables.

Twenty-one people, 11 men and 10 women, aged 65-82 years (mean 74, SD 4.9), with mean BMI of $27.1 \mathrm{~kg} / \mathrm{m}^{2}$ (SD 2.3 ) were recruited. When compared to a BMI of $27 \mathrm{~kg} / \mathrm{m}^{2}$ (considered normal in this population ${ }^{(5)}$ there was no significant difference. With the exception of women in relation to TSF there was no significant difference in, WC (cm), TSF (mm), AMC (cm) and HGD (kg) in comparison to normal values (WC: men mean 104.7, SD 9.8, women mean 87.6, SD 9.8, TSF: men mean 12.4, SD 4.5, women mean 18.9, SD 3.0, AMC: men mean 25.6, SD 2.6, women mean 23.4, SD 2.03, HGD men mean 38.0, sD 5.1, women mean 22.4, SD 5.1). HGD correlated with energy intake $(r=0.775, P<0.001)$ (see Figure).

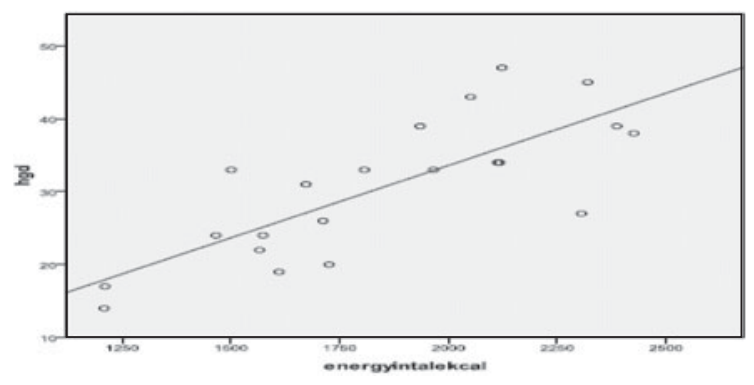

HGD also correlated with weight $(r=0.576, P<0.01)$, height $(r=0.656, P<0.01)$, WC $(r=0.66, P<0.01)$, TSF $(r=-0.562, P<0.01)$ and AMC $(r=0.465, P<0.05)$.

As HGD is a simple measure and an important marker in monitoring functional change and predicting functional decline the relationship between HGD and diet, particularly in relation to energy intake, it needs further investigation.

1. Gray A (2005) Population ageing and health care. Ageing Horizons (2), 15-20.

2. Gale CR, Martyn CN, Cooper C et al. (2007) Grip strength, body composition, and mortality. Int J Epidemiol 36, 228-235.

3. Giampaoli S, Ferrucci L, Cecchi F et al. (1999) Hand grip strength predicts incident disability in non-disabled older men. Age Ageing 28, 283-288.

4. Milne AC, Potter J, Vivanti A et al. (2009) Protein and energy supplementation in elderly people at risk from malnutrition. Cochrane Database Syst Rev, Issue 2.

5. Wynn A \& Wynn M (1995) What is a desirable weight for the elderly? Geriatric Med July Issue, 18. 